\title{
The Influence of Computer Screens on the Blinking Rate: A Comparison with and without a Screen
}

\author{
Charbel Faraj* \\ School of Advanced Research, Education and Accreditation (SAERA), Lebanon
}

Submission: April 28, 2020; Published: May 08, 2020

*Corresponding author: Charbel Faraj, School of Advanced Research, Education and Accreditation (SAERA), Lebanon

\begin{abstract}
Purpose: Ocular and visual symptoms are felt by many subjects during computer use. Previous studies have reported a reduced blink rate during computer operation and suggested that this may account for some of the symptoms, particularly dry eye. However, these earlier investigations did not include a control condition. To determine whether it is computer screen viewing that produces the change in blink rate, the present study compared blink patterns when reading from either a desktop computer monitor or a hard copy printed text under equivalent viewing conditions.
\end{abstract}

Keywords: Ocular; Visual symptoms; Blink rates; Computer vision; Diopter; Autorefractometer; Electronic screen; Phoropter, Schirmer's test strips, Fluorescein strips; Optotypes; Slit lamp; orthoptic problem

Methods: Subjects ( $\mathrm{N}=118$ ) were asked to undergo a continuous 20-minute reading task from either a desktop computer screen or a printed page at the same viewing distance. Identical text was used in the two sessions, with similar size and contrast. Target viewing angle and luminance were similar for the two conditions. Subjects were videotaped during the task to determine their blink rate. Immediately after the task, subjects completed a questionnaire regarding ocular symptoms experienced during the trial.

Results: Mean blink rates for the computer and hard copy conditions were $14.64 \& 13.12$ blinks per minute, respectively (p $<0.001)$. A significantly higher score of ocular symptoms was observed for the computer condition $(\mathrm{p}<0.001)$.

Discussion: Blink rates were not reduced during computer operation when compared with an equivalent hard copy control condition. Suggestions state that the previously observed differences in blink rate are more likely to be produced by changes in cognitive demand rather than the method of presentation. However, during the use of computers, a higher score of ocular symptoms was noted which may have been associated with visual tiredness.

Conclusion: The study aims to compare the rate of blinking with and without a screen, to know if screens cause an influence on the blinking rate. Results show the increase in the blinking rate is more remarkable with screen than without screen. Future studies must focus on increasing the blink amplitude to determine whether this provides a viable method of reducing symptoms during computer use.

\section{Introduction}

Our excessive and increasing use of computers has led to the development of a number of health problems. In contrast, our need for paper has decreased. Eye discomfort, fatigue, and stress are some of many work-related complaints and symptoms reported by many people who work on a computer. As computer use increases, so does the level of discomfort. The visual discomfort and related symptoms that occur in computer users should be treated as a growing health issue. Thus, arises the "Computer Vision Syndrome" which is the complex of eye and vision problems associated with experienced work while using the computer. A blink poses an interesting phenomenon that should be monitored and studied. In addition to being an important tool for scientific research, blinking is a necessity for our well-being, because of its role as a protective barrier for the eye and as a distributor of the tear film on the ocular surface. The blink rate can be defined as the frequency during which spontaneous blinks occur and can be even used as a measure of visual fatigue. Several studies have shown that computer screens directly influence the blinking rate.

Between 64 \& 90\% of computer users experience visual symptoms, which may include eyestrain, headaches, ocular discomfort, dry eye, diplopic, and blurred vision either at near or when looking into the distance after prolonged computer use according to Thomson [1]. A recent investigation of computer users in New York City noted that $40 \%$ of subjects reported tired eyes "at least half the time", whereas 32 \& 31\% reported dry eye and eye discomfort, respectively, with this same frequency 
[2]. Other studies have also found a strong association between dry eye and computer-related symptoms, with longer periods of computer work being associated with a higher prevalence of dry eye [3]. Investigations state that computer tasks reduce the blink rate [4-6]. Tsubota and Nakamori [4] compared the rate of blinking in 104 office workers when they were relaxed, reading a book, or viewing text on an electronic screen. Mean blink rates were 22 per minute while relaxed but only 10 per minute and 7 per minute when viewing the book or screen. These three testing conditions varied in the method of presentation and in task demand. It has been noted that as font size and contrast are reduced, blink rates are reduced as well [7] or the cognitive demand of the task increases $[8,9]$. Therefore, the differences observed by Tsubota and Nakamori [4] may be related to changes in task difficulty and are not the consequence of changing from printed material to electronic ones.

An important question arises: "Is the change in blink patterns during computer use related specifically to viewing the electronic screen or is it simply the result of performing a demanding nearvision task?"

Indeed, [10] states that both the visual nature of the task and the psychological status of the user should be considered in studies examining eye blink activity during computer use. Previous studies observing a reduced blink rate during computer use have not included an equivalent hard copy control trial, under the same viewing conditions. It has been suggested that the poorer image quality of the electronic screen may be responsible for the fluctuation in blink rate, when compared with printed materials. However, [7] observed that degrading the image quality by either inducing 1.00 diopter (D) of uncorrected astigmatism or presenting a target at only $7 \%$ contrast did not produce a significant change in blink rate for a given level of cognitive demand. Furthermore, [7] reported an increased blink rate when induced refractive error, glare, reduced contrast, and accommodative stress (varying the accommodative stimulus by \pm 1.50 D during the course of the task). In addition, [11] found that a significant reduction in blink rate is observed during the introduction of an antireflection film over a computer monitor to reduce glare produced. Since evidence lacks regarding the role of the electronic screen in the change in blinking rates, the aim of the present study was to determine if a difference in blink patterns exists when subjects perform similar reading tasks from either a computer monitor or printed materials.

\section{Methods \& Materials}

The study involved 118 patients aged between 18 \& 35 years. They were examined in an optometry clinic. Subjects included were those having a monocular visual acuity at least $10 / 10$ for far and near, with or without optical correction. Subjects excluded were those having an uncorrected ametropia, having a squint or an orthoptic problem, having an eye pathology or dry eye, having undergone a previous eye surgery, patients suffering from a general pathology influencing the visual system such as diabetes, high blood pressure, etc.

The examination room was equipped with a slit lamp, an autorefractometer, a digital phoropter, Schirmer's test strips, fluorescein strips, a projector of optotypes, a laptop, printed papers and a video camera. The recruited patient was received in a quiet room, with moderate lighting, of moderate temperature, without ventilation or any other factor that could hinder the examination of tear secretion and refraction. The contrast on the computer screen and the printed paper was the same. he text read had the following characteristics identical on both computer and paper: Size 14, Font Times New Roman, Black color, a single space between words. Subjects wearing contact lenses got rid of their lenses before 48 hours of our experience. Subjects were not informed that their blinks were being monitored or recorded as this could cause voluntary blinks. All of the subjects were interviewed to fill out their examination sheets to ensure first of all their age and the absence of any ophthalmological and general history.

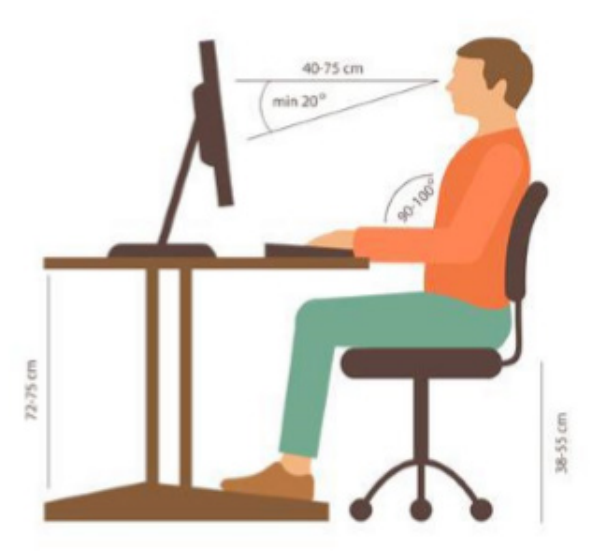

Figure 1: Position of the subject during the 2 sessions. 
We started with the study of lacrimal secretion with the Schirmer test (normal if more than $15 \mathrm{~mm}$ per 5 minutes) and the B.U.T. (normal if break after 10 seconds). Then, we performed orthoptic tests including the motility test, PPC (Punctum Proximum Convergence), cover/uncover test. These tests must be within the normal limits. Visual acuity from far and near, raw and with correction, was measured monocularly and binocularly. It must be at least $10 / 10$. To ensure that the patient is emmetropic or perfectly corrected, we performed refraction. It was preceded by a measurement on the autorefractometer. After completing the information and carrying out the tests mentioned above, we made the experience concrete, divided into two phases of 20 minutes each, and separated by an interval of at least 24 hours.

The patient read identical text on a laptop screen during the first phase and on printed paper during the second phase. During these two phases, the subjects' eyes were filmed using a video camera from a cell phone (smartphone), held by a support. Sufficient material was provided for 20 minutes of reading without repetition. The recorded videos allowed us to count the number of blinks during each phase. The results of the number of blinks enabled us to determine the blink rate per minute. Immediately after completion of each reading task, subjects completed a written questionnaire asking about their level of ocular symptoms observed during the two sessions. The results were recorded on a scale ranging from 0-10 (0: zero, 10: severe).

\section{Statistical test:}

The statistical test adapted in the study is the "analysis of variance" as known as ANOVA. It is a test that is applied when one or more discrete explanatory variables (the factors) that influence the distribution of a variable continue to be explained. We can compare Sig (The degree of significance) with $\alpha=5 \%$ (The risk of error). If $\mathrm{Sig}<\alpha$ The qualitative variable has an effect on the quantitative variable.

\section{Result}

\section{Population Study}

This part includes a descriptive presentation of the variables studied throughout this study.

We considered 118 patients of an age group [18-35], taking into account the theoretical increase in dry eyes, the decrease in the blinking rate and the appearance of presbyopia with age. The average age is 26.72 years with a standard deviation of 4.563 . The distribution of the population by gender was $56.78 \%$ male and $43.22 \%$ female. $66.90 \%$ were emmetropes and $33.10 \%$ were corrected ametropes [Tables 1-8]
Table 1: Distribution of the average blink rate without screen by gender, profession and refractive state.

\begin{tabular}{|c|c|c|c|}
\hline \multicolumn{2}{|c|}{ Without Screen } & $\mathbf{N}$ & Average \\
\hline \multirow{9}{*}{ By gender } & Male & 67 & 13.1701 \\
\hline & Female & 51 & 13.0451 \\
\hline & Total & 118 & 13.1161 \\
\hline & Students & 17 & 13.5176 \\
\hline & Teachers & 16 & 12.975 \\
\hline & CEOs & 10 & 12.85 \\
\hline & Architects & 4 & 13.025 \\
\hline & Biologists & 3 & 13.5667 \\
\hline & Engineers & 4 & 12.125 \\
\hline \multirow{8}{*}{ By profession } & Translators & 3 & 12.2333 \\
\hline & Pharmacists & 7 & 13.3429 \\
\hline & Salesmen & 3 & 13.1 \\
\hline & Waiters & 7 & 13.6286 \\
\hline & Office workers & 8 & 12.8375 \\
\hline & Others & 36 & 13.1389 \\
\hline & Total & 118 & 13.1161 \\
\hline & Emmetrope & 79 & 13.1215 \\
\hline \multirow[t]{2}{*}{$\begin{array}{c}\text { By refractive } \\
\text { state }\end{array}$} & Ametrope & 39 & 13.1051 \\
\hline & Total & 118 & 13.1161 \\
\hline
\end{tabular}

Table 2: Distribution of the average blinking rate with screen by gender, profession and refractive state.

\begin{tabular}{|c|c|c|c|}
\hline \multicolumn{2}{|c|}{ Whith Screen } & N & Average \\
\hline \multirow{4}{*}{ By gender } & Male & 67 & 14.8239 \\
\cline { 2 - 4 } & Female & 51 & 14.3941 \\
\cline { 2 - 4 } & Total & 118 & 14.6381 \\
\cline { 2 - 4 } & Students & 17 & 14.8882 \\
\cline { 2 - 4 } & Teachers & 16 & 14.6938 \\
\cline { 2 - 4 } & CEOs & 10 & 14.4 \\
\cline { 2 - 4 } & Architects & 4 & 13.8 \\
\cline { 2 - 4 } & Biologists & 3 & 14.7333 \\
\cline { 2 - 4 } & Engineers & 4 & 14.175 \\
\hline
\end{tabular}




\begin{tabular}{|c|c|c|c|}
\hline \multirow{7}{*}{ By profession } & Translators & 3 & 12.5667 \\
\cline { 2 - 4 } & Pharmacists & 7 & 14.5714 \\
\cline { 2 - 4 } & Salesmen & 3 & 14.8 \\
\cline { 2 - 4 } & Waiters & 7 & 15.1 \\
\cline { 2 - 4 } & Office workers & 8 & 15.5125 \\
\cline { 2 - 4 } & Others & 36 & 14.5861 \\
\cline { 2 - 4 } & Total & 118 & 14.6381 \\
\cline { 2 - 4 } & Emmetrope & 79 & 14.5038 \\
\hline By refractive state & Ametrope & 39 & 14.9103 \\
\hline & Total & 118 & 14.6381 \\
\hline
\end{tabular}

Table 3: Distribution of the population by gender.

\begin{tabular}{|c|c|c|}
\hline Gender & Frequency & Percentage \\
\hline Male & 67 & $56.78 \%$ \\
\hline Female & 51 & $43.22 \%$ \\
\hline Total & 118 & $100 \%$ \\
\hline
\end{tabular}

Table 4: Distribution of the population by profession.

\begin{tabular}{|c|c|c|}
\hline Profession & Frequency & Percentage \\
\hline Students & 17 & $14.41 \%$ \\
\hline Teachers & 16 & $13.56 \%$ \\
\hline Businessmen & 10 & $8.47 \%$ \\
\hline Architects & 4 & $3.39 \%$ \\
\hline Biologists & 3 & $2.54 \%$ \\
\hline Engineers & 4 & $3.39 \%$ \\
\hline Translators & 3 & $2.54 \%$ \\
\hline Pharmacists & 7 & $5.93 \%$ \\
\hline Sellers & 3 & $2.54 \%$ \\
\hline Waiters & 7 & $5.93 \%$ \\
\hline Office workers & 8 & $6.78 \%$ \\
\hline Others & 36 & $30.51 \%$ \\
\hline Total & 118 & $100 \%$ \\
\hline
\end{tabular}

Table 5: Distribution of the population by refractive state.

\begin{tabular}{|c|c|c|}
\hline Refractive state & Frequency & Percentage \\
\hline Emmetrope & 79 & $66.90 \%$ \\
\hline Corrected ametrope & 39 & $33.10 \%$ \\
\hline Total & 118 & $100 \%$ \\
\hline
\end{tabular}

Table 6: Comparison of symptom score with and without screen.

\begin{tabular}{|c|c|c|c|c|c|}
\hline Score over 10 & $\begin{array}{c}\text { Score over } \\
10\end{array}$ & & Without screen & With screen & Total \\
\hline \multirow{8}{*}{$\begin{array}{c}\text { Blurred vision while } \\
\text { looking at text }\end{array}$} & \multirow{2}{*}{0} & Frequency & 70 & 40 & 110 \\
\hline & & Percentage & $59.30 \%$ & $33.90 \%$ & $46.60 \%$ \\
\hline & \multirow{2}{*}{1} & Frequency & 48 & 27 & 75 \\
\hline & & Percentage & $40.70 \%$ & $22.90 \%$ & $31.80 \%$ \\
\hline & \multirow{2}{*}{2} & Frequency & 0 & 37 & 37 \\
\hline & & Percentage & $0.00 \%$ & $31.40 \%$ & $15.70 \%$ \\
\hline & \multirow{2}{*}{3} & Frequency & 0 & 14 & 14 \\
\hline & & Percentage & $0.00 \%$ & $11.90 \%$ & $5.90 \%$ \\
\hline \multirow{10}{*}{$\begin{array}{l}\text { Blurred vision looking } \\
\text { from afar at the end of } \\
\text { the task up close }\end{array}$} & \multirow{2}{*}{0} & Frequency & 76 & 53 & 129 \\
\hline & & Percentage & $64.40 \%$ & $44.90 \%$ & $54.70 \%$ \\
\hline & \multirow{2}{*}{1} & Frequency & 42 & 59 & 101 \\
\hline & & Percentage & $35.60 \%$ & $50.00 \%$ & $42.80 \%$ \\
\hline & \multirow{2}{*}{2} & Frequency & 0 & 4 & 4 \\
\hline & & Percentage & $0.00 \%$ & $3.40 \%$ & $1.70 \%$ \\
\hline & \multirow{2}{*}{3} & Frequency & 0 & 1 & 1 \\
\hline & & Percentage & $0.00 \%$ & $0.80 \%$ & $0.40 \%$ \\
\hline & \multirow{2}{*}{4} & Frequency & 0 & 1 & 1 \\
\hline & & Percentage & $0.00 \%$ & $0.80 \%$ & $0.40 \%$ \\
\hline
\end{tabular}


JOJ Ophthalmology

\begin{tabular}{|c|c|c|c|c|c|}
\hline \multirow{10}{*}{$\begin{array}{l}\text { Difficulty / slowness } \\
\text { in refocusing eyes } \\
\text { from one distance to } \\
\text { another: }\end{array}$} & \multirow{2}{*}{0} & Frequency & 77 & 54 & 131 \\
\hline & & Percentage & $65.30 \%$ & $45.80 \%$ & $55.50 \%$ \\
\hline & \multirow{2}{*}{1} & Frequency & 41 & 50 & 91 \\
\hline & & Percentage & $34.70 \%$ & $42.40 \%$ & $38.60 \%$ \\
\hline & \multirow{2}{*}{2} & Frequency & 0 & 8 & 8 \\
\hline & & Percentage & $0.00 \%$ & $6.80 \%$ & $3.40 \%$ \\
\hline & \multirow{2}{*}{3} & Frequency & 0 & 4 & 4 \\
\hline & & Percentage & $0.00 \%$ & $3.40 \%$ & $1.70 \%$ \\
\hline & \multirow{2}{*}{4} & Frequency & 0 & 2 & 2 \\
\hline & & Percentage & $0.00 \%$ & $1.70 \%$ & $0.80 \%$ \\
\hline \multirow{16}{*}{ Irritated or burning eyes } & \multirow{2}{*}{0} & Frequency & 52 & 30 & 82 \\
\hline & & Percentage & $44.10 \%$ & $25.40 \%$ & $34.70 \%$ \\
\hline & \multirow{2}{*}{1} & Frequency & 52 & 6 & 58 \\
\hline & & Percentage & $44.10 \%$ & $5.10 \%$ & $24.60 \%$ \\
\hline & \multirow{2}{*}{2} & Frequency & 12 & 41 & 53 \\
\hline & & Percentage & $10.20 \%$ & $34.70 \%$ & $22.50 \%$ \\
\hline & \multirow{2}{*}{3} & Frequency & 0 & 30 & 30 \\
\hline & & Percentage & $0.00 \%$ & $25.40 \%$ & $12.70 \%$ \\
\hline & \multirow{2}{*}{4} & Frequency & 0.00 & 800.00 & 800.00 \\
\hline & & Percentage & $0.00 \%$ & $6.80 \%$ & $3.40 \%$ \\
\hline & \multirow{2}{*}{5} & Frequency & 100.00 & 100.00 & 200.00 \\
\hline & & Percentage & $0.80 \%$ & $0.80 \%$ & $0.80 \%$ \\
\hline & \multirow{2}{*}{6} & Frequency & 100.00 & 100.00 & 200.00 \\
\hline & & Percentage & $0.80 \%$ & $0.80 \%$ & $0.80 \%$ \\
\hline & \multirow{2}{*}{7} & Frequency & 0.00 & 100.00 & 100.00 \\
\hline & & Percentage & $0.00 \%$ & $0.80 \%$ & $0.40 \%$ \\
\hline \multirow{18}{*}{ Dry eyes } & \multirow{2}{*}{0} & Frequency & 3200.00 & 600.00 & 3800.00 \\
\hline & & Percentage & $27.10 \%$ & $5.10 \%$ & $16.10 \%$ \\
\hline & \multirow{2}{*}{1} & Frequency & 6100.00 & 1400.00 & 7500.00 \\
\hline & & Percentage & $51.70 \%$ & $11.90 \%$ & $31.80 \%$ \\
\hline & \multirow{2}{*}{2} & Frequency & 2300.00 & 2200.00 & 4500.00 \\
\hline & & Percentage & $19.50 \%$ & $18.60 \%$ & $19.10 \%$ \\
\hline & \multirow{2}{*}{3} & Frequency & 0.00 & 3100.00 & 3100.00 \\
\hline & & Percentage & $0.00 \%$ & $26.30 \%$ & $13.10 \%$ \\
\hline & \multirow{2}{*}{4} & Frequency & 100.00 & 3300.00 & 3400.00 \\
\hline & & Percentage & $0.80 \%$ & $28.00 \%$ & $14.40 \%$ \\
\hline & \multirow{2}{*}{5} & Frequency & 0.00 & 500.00 & 500.00 \\
\hline & & Percentage & $0.00 \%$ & $4.20 \%$ & $2.10 \%$ \\
\hline & \multirow{2}{*}{6} & Frequency & 0.00 & 600.00 & 600.00 \\
\hline & & Percentage & $0.00 \%$ & $5.10 \%$ & $2.50 \%$ \\
\hline & \multirow{2}{*}{7} & Frequency & 100.00 & 0.00 & 100.00 \\
\hline & & Percentage & $0.80 \%$ & $0.00 \%$ & $0.40 \%$ \\
\hline & 0 & Frequency & 0.00 & 100.00 & 100.00 \\
\hline & 8 & Percentage & $0.00 \%$ & $0.80 \%$ & $0.40 \%$ \\
\hline
\end{tabular}


JOJ Ophthalmology

\begin{tabular}{|c|c|c|c|c|c|}
\hline \multirow{11}{*}{ Eye fatigue } & \multirow{2}{*}{0} & Frequency & 2900.00 & 400.00 & 3300.00 \\
\hline & & Percentage & $24.60 \%$ & $3.40 \%$ & $14.00 \%$ \\
\hline & 1 & Frequency & 6800.00 & 1000.00 & 7800.00 \\
\hline & & Percentage & $57.60 \%$ & $8.50 \%$ & $33.10 \%$ \\
\hline & 2 & Frequency & 1400.00 & 1900.00 & 3300.00 \\
\hline & & Percentage & $11.90 \%$ & $16.10 \%$ & $14.00 \%$ \\
\hline & & Frequency & 500.00 & 3000.00 & 3500.00 \\
\hline & 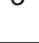 & Percentage & $4.20 \%$ & $25.40 \%$ & $14.80 \%$ \\
\hline & & Frequency & 100.00 & 3300.00 & 3400.00 \\
\hline & $T$ & Percentage & $0.80 \%$ & $28.00 \%$ & $14.40 \%$ \\
\hline & 5 & Frequency & 0.00 & $1400.00 \%$ & $1400.00 \%$ \\
\hline \multirow{18}{*}{ Eye discomfort } & \multirow{2}{*}{0} & Frequency & $34 \%$ & $5 \%$ & $39 \%$ \\
\hline & & Percentage & $28.80 \%$ & $4.20 \%$ & $16.50 \%$ \\
\hline & \multirow{2}{*}{1} & Frequency & 57 & 9 & 66 \\
\hline & & Percentage & $48.30 \%$ & $7.60 \%$ & $28.00 \%$ \\
\hline & \multirow{2}{*}{2} & Frequency & 21 & 16 & 37 \\
\hline & & Percentage & $17.80 \%$ & $13.60 \%$ & $15.70 \%$ \\
\hline & \multirow{2}{*}{3} & Frequency & 4 & 42 & 46 \\
\hline & & Percentage & $3.40 \%$ & $35.60 \%$ & $19.50 \%$ \\
\hline & \multirow{2}{*}{4} & Frequency & 0 & 28 & 28 \\
\hline & & Percentage & $0.00 \%$ & $23.70 \%$ & $11.90 \%$ \\
\hline & \multirow{2}{*}{5} & Frequency & 1 & 15 & 16 \\
\hline & & Percentage & $0.80 \%$ & $12.70 \%$ & $6.80 \%$ \\
\hline & \multirow{2}{*}{6} & Frequency & 0 & 1 & 1 \\
\hline & & Percentage & $0.00 \%$ & $0.80 \%$ & $0.40 \%$ \\
\hline & 7 & Frequency & 1 & 1 & 2 \\
\hline & $T$ & Percentage & $0.80 \%$ & $0.80 \%$ & $0.80 \%$ \\
\hline & 8 & Frequency & 0 & 1 & 1 \\
\hline & 0 & Percentage & $0.00 \%$ & $0.80 \%$ & $0.40 \%$ \\
\hline \multirow{10}{*}{ Photophobia } & \multirow{2}{*}{0} & Frequency & 92 & 56 & 148 \\
\hline & & Percentage & $78.00 \%$ & $47.50 \%$ & $62.70 \%$ \\
\hline & \multirow{2}{*}{1} & Frequency & 26 & 38 & 64 \\
\hline & & Percentage & $22.00 \%$ & $32.20 \%$ & $27.10 \%$ \\
\hline & \multirow{2}{*}{2} & Frequency & 0 & 21 & 21 \\
\hline & & Percentage & $0.00 \%$ & $17.80 \%$ & $8.90 \%$ \\
\hline & \multirow{2}{*}{3} & Frequency & 0 & 2 & 2 \\
\hline & & Percentage & $0.00 \%$ & $1.70 \%$ & $0.80 \%$ \\
\hline & 5 & Frequency & 0 & 1 & 1 \\
\hline & 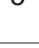 & Percentage & $0.00 \%$ & $0.80 \%$ & $0.40 \%$ \\
\hline \multirow{8}{*}{ Headache } & \multirow{2}{*}{0} & Frequency & 74 & 55 & 12 \\
\hline & & Percentage & $62.70 \%$ & $46.60 \%$ & $54.70 \%$ \\
\hline & \multirow{2}{*}{1} & Frequency & 41 & 28 & 69 \\
\hline & & Percentage & $34.70 \%$ & $23.70 \%$ & $29.20 \%$ \\
\hline & \multirow{2}{*}{2} & Frequency & 3 & 34 & 37 \\
\hline & & Percentage & $2.50 \%$ & $28.80 \%$ & $15.70 \%$ \\
\hline & \multirow{2}{*}{4} & Frequency & 0 & 1 & 1 \\
\hline & & Percentage & $0.00 \%$ & $0.80 \%$ & $0.40 \%$ \\
\hline
\end{tabular}


JOJ Ophthalmology

\begin{tabular}{|c|c|c|c|c|}
\hline \multirow{2}{*}{$\begin{array}{c}\text { Total(for every } \\
\text { symptom) }\end{array}$} & Frequency & 118 & 118 \\
\cline { 2 - 5 } & & Percentage & $100.00 \%$ & $100.00 \%$ \\
\hline
\end{tabular}

Table 7: Comparison of average score for each symptom with and without screen.

\begin{tabular}{|c|c|c|c|}
\hline \multicolumn{2}{|l|}{ Symptom } & \multirow{2}{*}{$\frac{\mathbf{N}}{118}$} & \multirow{2}{*}{$\begin{array}{c}\text { Average score } \\
0.41\end{array}$} \\
\hline Plunrod uricion unbilo lo lring tont & Without Screen & & \\
\hline Biurrea vision wnile looking at text & With Screen & 118 & 1.21 \\
\hline \multirow{2}{*}{$\begin{array}{l}\text { Blurred vision looking } \\
\text { from afar at the end of the task up close }\end{array}$} & Without Screen & 118 & 0.36 \\
\hline & With Screen & 118 & 0.78 \\
\hline \multirow{2}{*}{$\begin{array}{l}\text { Difficulty / slowness in } \\
\text { refocusing eyes from one distance to another }\end{array}$} & Without Screen & 118 & 0.35 \\
\hline & With Screen & 118 & 0.73 \\
\hline \multirow{2}{*}{ Irritated or burning eyes } & Without Screen & 118 & 0.75 \\
\hline & With Screen & 118 & 1.95 \\
\hline \multirow{2}{*}{ Dry eyes } & Without Screen & 118 & 1 \\
\hline & With Screen & 118 & 2.98 \\
\hline \multirow{2}{*}{ Eye fatigue } & Without Screen & 118 & 1.03 \\
\hline & With Screen & 118 & 3.29 \\
\hline \multirow{2}{*}{ Eye discomfort } & Without Screen & 118 & 1.04 \\
\hline & With Screen & 118 & 3.18 \\
\hline \multirow{2}{*}{ Photophobia } & Without Screen & 118 & 0.22 \\
\hline & With Screen & 118 & 0.77 \\
\hline \multirow{2}{*}{ Headache } & Without Screen & 118 & 0.4 \\
\hline & With Screen & 118 & 0.85 \\
\hline
\end{tabular}

Table 8: Distribution of the number of blinking per minute with and without screen among participants.

\begin{tabular}{|c|c|c|c|c|}
\hline \multirow{2}{*}{ Number of blinks / min } & \multicolumn{2}{|c|}{ Without screen } & \multicolumn{2}{|c|}{ With screen } \\
\hline & Frequency & Percentage & Frequency & Percentage \\
\hline 10 & & & 1 & $0.85 \%$ \\
\hline 10.1 & 1 & $0.85 \%$ & & \\
\hline 10.9 & 1 & $0.85 \%$ & 1 & $0.85 \%$ \\
\hline 11.3 & 1 & $0.85 \%$ & & \\
\hline 11.4 & 2 & $1.69 \%$ & & \\
\hline 11.5 & 2 & $1.69 \%$ & & \\
\hline 11.7 & 5 & $4.24 \%$ & & \\
\hline 11.8 & 4 & $3.39 \%$ & 1 & $0.85 \%$ \\
\hline 11.9 & 1 & $0.85 \%$ & 1 & $0.85 \%$ \\
\hline 12 & 1 & $0.85 \%$ & & \\
\hline 12.1 & 3 & $2.54 \%$ & & \\
\hline 12.2 & 4 & $3.39 \%$ & & \\
\hline 12.3 & 9 & $7.63 \%$ & 1 & $0.85 \%$ \\
\hline 12.4 & 1 & $0.85 \%$ & 2 & $1.69 \%$ \\
\hline 12.5 & 2 & $1.69 \%$ & & \\
\hline 12.6 & 1 & $0.85 \%$ & & \\
\hline 12.7 & 2 & $1.69 \%$ & 1 & $0.85 \%$ \\
\hline
\end{tabular}


JOJ Ophthalmology

\begin{tabular}{|c|c|c|c|c|}
\hline 12.8 & 8 & $6.78 \%$ & & \\
\hline 12.9 & 6 & $5.08 \%$ & 4 & $3.39 \%$ \\
\hline 13 & 6 & $5.08 \%$ & 1 & $0.85 \%$ \\
\hline 13.1 & 5 & $4.24 \%$ & 1 & $0.85 \%$ \\
\hline 13.2 & 9 & $7.63 \%$ & 2 & $1.69 \%$ \\
\hline 13.3 & 6 & $5.08 \%$ & & \\
\hline 13.4 & 2 & $1.69 \%$ & 3 & $2.54 \%$ \\
\hline 13.5 & & & 1 & $0.85 \%$ \\
\hline 13.6 & 1 & $0.85 \%$ & & \\
\hline 13.7 & 1 & $0.85 \%$ & & \\
\hline 13.8 & 6 & $5.08 \%$ & 4 & $3.39 \%$ \\
\hline 13.9 & 2 & $1.69 \%$ & 2 & $1.69 \%$ \\
\hline 14 & 4 & $3.39 \%$ & 5 & $4.24 \%$ \\
\hline 14.1 & 5 & $4.24 \%$ & 4 & $3.39 \%$ \\
\hline 14.2 & 4 & $3.39 \%$ & 7 & $5.93 \%$ \\
\hline 14.3 & 4 & $3.39 \%$ & 5 & $4.24 \%$ \\
\hline 14.4 & & & 7 & $5.93 \%$ \\
\hline 14.5 & & & 4 & $3.39 \%$ \\
\hline 14.7 & 2 & $1.69 \%$ & 2 & $1.69 \%$ \\
\hline 14.8 & & & 4 & $3.39 \%$ \\
\hline 14.9 & & & 1 & $0.85 \%$ \\
\hline 15 & & & 5 & $4.24 \%$ \\
\hline 15.1 & 1 & $0.85 \%$ & 3 & $2.54 \%$ \\
\hline 15.2 & 1 & $0.85 \%$ & 8 & $6.78 \%$ \\
\hline 15.3 & 1 & $0.85 \%$ & 9 & $7.63 \%$ \\
\hline 15.4 & & & 1 & $0.85 \%$ \\
\hline 15.5 & & & 2 & $1.69 \%$ \\
\hline 15.6 & & & 1 & $0.85 \%$ \\
\hline 15.7 & & & 5 & $4.24 \%$ \\
\hline 15.8 & & & 2 & $1.69 \%$ \\
\hline 16 & & & 4 & $3.39 \%$ \\
\hline 16.1 & 1 & $0.85 \%$ & 1 & $0.85 \%$ \\
\hline 16.2 & 1 & $0.85 \%$ & 3 & $2.54 \%$ \\
\hline 16.3 & & & 1 & $0.85 \%$ \\
\hline 16.5 & & & 2 & $1.69 \%$ \\
\hline 16.7 & & & 2 & $1.69 \%$ \\
\hline 17 & & & 1 & $0.85 \%$ \\
\hline 17.1 & & & 1 & $0.85 \%$ \\
\hline 17.2 & 2 & $1.69 \%$ & & \\
\hline 17.4 & & & 1 & $0.85 \%$ \\
\hline 17.9 & & & 1 & $0.85 \%$ \\
\hline
\end{tabular}


Figure 2: Comparison of average score for each symptom with and without screen.

Figure 3:Distribution of the number of blinking per minute with and without screen among participants.

\section{Results}

\section{Without screen:}

Males and females are almost equal.

P-value $=0.563>0.05$

The variations are slight between all professions.

P-value $=0.534>0.05$

The 2 refractive state groups are almost equal.

P-value $=0.943>0.05$

\section{With screen:}

The majority of the subjects are males.

P-value $=0.07>0.05$

The majority are indoor workers.

$\mathrm{P}$-value $=0.135>0.05$
The majority are perfectly corrected ametropics.

P-value $=0.104>0.0$

\section{Symptoms}

The ANOVA test carried out between the symptoms and the experience "without screen" or "with screen" shows us that there is a relationship between these two variables P-value $\leq 0.05$. The majority of subjects without a screen have a rate of 13.20 and subjects with a screen have a rate of 15.30. The average blink rate without and with screen are respectively 13.1161 and 14.6381. This means, as shown in the diagram, that the average blinking rate with screen is slightly higher than that without screen. The ANOVA test shows us a significant variation P-value $0.00<0.05$.

\section{Results Analysis}

The aim of this research was to study the influence of computer screens on the rate of blinking and the symptoms observed. This was based on a comparison of the results obtained by reading an identical text on a screen and on a printed paper, 
since the influence of the screens causes a variation in the rate of blinking and the appearance of symptoms. This work was carried out on 118 subjects aged between 18 \& 35, average is 26.72. $66.90 \%$ were emmetropes against $33.10 \%$ ametropes perfectly corrected? Thus, this margin of difference allowed us to establish a comparative study between the emmetropes and the ametropes perfectly corrected. All patients underwent the test with and without a screen. The average blink rate among emmetropics was 13.12 without screen versus 14.50 with screen. Whereas in perfectly corrected ametropics, the average was 13.10 without screen against 14.91 with screen. Fifty-six-point seventyeight percent of the subjects were males and $43.22 \%$ were females. Thus, this margin of difference allowed us to establish a comparative study between males and females. All patients underwent the test with and without a screen.

The average blink rate for males was 13.17 without screen versus 14.82 with screen. Whereas in females, the average was 13.04 without screen against 14.39 with screen. By studying the significance ( $\mathrm{p}$-value) without screen according to sex ( $\mathrm{p}$-value $=0.563>0.05)$, the refractive state $(p$-value $=0.943>0.05)$ and the profession ( $\mathrm{p}$-value $=0.534>0.05$ ), we conclude that the variations are not significant. On the other hand, by studying the significance ( $\mathrm{p}$-value) with screen according to sex ( $\mathrm{p}$-value = $0.07>0.05)$, the refractive state $(p$-value $=0.104>0.05)$ and the profession ( $p$-value $=0.135>0.05$ ), we deduce that the variations are also non-significant.

Compared to the symptoms found without a screen, we note blurred vision scores while looking at text 0.41 , blurred vision looking from afar at the end of the task up close 0.36 , difficulty / slowness in refocusing eyes from one distance to another 0.35 , irritated or burning eyes 0.75 , dry eyes 1.00 , eye fatigue 1.03 , eye discomfort 1.04, photophobia 0.22 and headache 0.40 . On the other hand, we find with screen an increase in the scores of all the symptoms (Figure 2): blurred vision while looking at text 1.21, blurred vision by looking from afar at the end of the task near 0.78 , difficulty / slowness in refocusing eyes from one distance to another 0.73 , irritated or burning eyes 1.95 , dry eyes 2.98 , tired eyes 3.29 , eye discomfort 3.18 , photophobia 0.77 and headache 0.85 . The ANOVA statistical test shows a significant variation ( $p$-value $<0.05$ ) for the 9 symptoms. The variation of the average blink rate with screen is 14.63 , greater than that without the value 13.11 screen. There is a significant variation according to ANOVA, p-value $=0.00<0.05$.

\section{Discussion}

The total symptom score was higher regarding the computer condition. However, between the 2 presentation methods, the overall blink rate was not notably different. The absence of a reduction in blink rate with computer use is contrary to previous reports [4-6]. As noted earlier, these previous investigations did not include an equivalent hard copy control trial. In the present study, the hard copy condition was performed at the same viewing distance and gaze angle and comprised the same reading material while both target contrast and letter size were matched as closely as possible. Since no difference was noted between the two conditions, this would imply that the differences reported previously were caused by variations in task demand rather than being produced directly by the computer monitor. For example, [4] found a significantly lower rate of blinking in office workers viewing text on an electronic screen compared with when they were relaxed. [13]. observed a reduced blink rate when the subjects were performing a computer task compared with "general conversation". In all cases, the increased cognitive demand of the computer task could account for the change in blink rate reported previously. Accordingly, evidence lacks to support the proposal that performing a task using an electronic monitor will produce a change in blink rate compared to carrying out the same task using printed material.

A review by Doughty [2] noted environmental conditions affect the blink rate (e.g., ambient temperature and humidity), gaze angle, and verbalization. Although the environmental conditions were not strictly under control, all of the experimental sessions were conducted in the same clinic, with variations in temperature or humidity levels. Both gaze angle and verbalization (i.e., the requirement to read the text aloud) were the same for both conditions. Doughty [2] suggested that reading aloud (as opposed to sitting in silence) might represent a "subtle stimulus" to blinks, so one might conclude that slightly different results could be reached if the subject read silently which is the popular case in work environments. Although verbalization has been shown to increase the blink rate, [12,13], moving the jaw on its own does not significantly affect the blink rate [14]. Accordingly, task difficulty may form some sort of relationship between blink rate and speech [14-18]. It seems reasonable to assume that, no significant difference in blink rates between the computer and hard copy conditions would have been noted even if the subjects had read silently rather than aloud.

\section{Conclusion}

The aim of this study is to compare the rate of blinking with and without a screen, in order to know if screens cause an influence on the blinking rate and on the symptoms related. According to the results, the increase in the blinking rate is more remarkable with screen than without screen. By focusing on the symptoms observed, we notice an increase of the scores with the computer screen. The difference observed can be attributed to the demand for the reading task, which is more complex on the computer screen than on paper.

This allows us to conclude that the symptoms present a more severe score on the computer screen, as well as a slight increase in the rate of blinking.

It shows that under tested conditions, no significant change in blink rate is observed when using computers exclusively. The level 
of cognitive demand is likely responsible for blink rate decline. As mentioned before, increased symptoms during computer operation were associated with a higher percentage of incomplete blinks, future studies must focus on increasing the blink amplitude to determine whether this provides a viable method of reducing symptoms during computer use.

\section{References}

1. Thomson WD (1998) Eye problems and visual display terminals--the facts and the fallacies. Ophthalmic and Physiological Optics 18(2): 111119.

2. Portello JK, Rosenfield M, Bababekova Y, Estrada JM, Leon A (2012) Computer-related visual symptoms in office workers. Ophthalmic \& physiological optics: the journal of the British College of Ophthalmic Opticians (Optometrists) 32(5): 375-382.

3. Uchino M, Schaumberg D, Dogru M, Uchino Y, Fukagawa K, et al. (2008) Prevalence of Dry Eye Disease among Japanese Visual Display Terminal Users. Ophthalmology 115(11): 1982-1988.

4. Tsubota K, Nakamori K (1993) Dry Eyes and Video Display Terminals. New England Journal Of Medicine 328(8): 584-584.

5. Patel S, Henderson R, Bradley L, Galloway B, Hunter L (1991) Effect of Visual Display Unit Use on Blink Rate and Tear Stability. Optometry And Vision Science 68(11): 888-892.

6. Schlote T, Kadner G, Freudenthaler N (2004) Marked reduction and distinct patterns of eye blinking in patients with moderately dry eyes during video display terminal use. Graefe's Archive For Clinical And Experimental Ophthalmology 242(4): 306-312.

7. Gowrisankaran S, Nahar N, Hayes J, Sheedy J (2012) Asthenopia and Blink Rate Under Visual and Cognitive Loads. Optometry And Vision Science 89(1): 97-104.

8. Himebaugh N, Begley C, Bradley A, Wilkinson J (2009) Blinking and Tear Break-Up During Four Visual Tasks. Optometry And Vision Science: 86(2): E106-E114.
9. Jansen M, Begley C, Himebaugh N, Port N (2010) Effect of Contact Lens Wear and a Near Task on Tear Film Break-Up. Optometry And Vision Science 87(5): 350-357.

10. Doughty M (2001) Consideration of Three Types of Spontaneous Eyeblink Activity in Normal Humans: during Reading and Video Display Terminal Use, in Primary Gaze, and while in Conversation. Optometry And Vision Science 78(10): 712-725.

11. Miyake-Kashima M, Dogru M, Nojima T, Murase M, Matsumoto $Y$, et al. (2005) The Effect of Antireflection Film Use on Blink Rate and Asthenopic Symptoms during Visual Display Terminal Work. Cornea 24(5): 567-570.

12. Hall A (1945) The Origin and Purposes of Blinking. British Journal Of Ophthalmology 29(9): 445-467.

13. Schuri U, Cramon D (1981) Heart Rate and Blink Rate Responses During Mental Arithmetic With and Without Continuous Verbalization of Results. Psychophysiology 18(6): 650-653.

14. Karson C, Berman K, Donnelly E, Mendelson W, Kleinma J, Wyatt R (1981) Speaking, thinking, and blinking. Psychiatry Research 5(3): 243-246.

15. Chu C, Rosenfield M, Portello JK, Benzoni JA, Collier JD (2011) A comparison of symptoms after viewing text on a computer screen and hardcopy. Ophthalmic \& physiological optics : the journal of the British College of Ophthalmic Opticians (Optometrists).31(1), 29-32.

16. Gowrisankaran S, Sheedy J, Hayes J (2007) Eyelid Squint Response to Asthenopia-Inducing Conditions. Optometry And Vision Science 84(7): 611-619.

17. Portello JK, Rosenfield M, Chu C (2013) Blink Rate, Incomplete Blinks and Computer Vision Syndrome. Optometry And Vision Science 90(5): 482-487.

18. Von Cramon D, Schuri U (1980) Blink frequency and speech motor activity. Neuropsychologia 18(4-5): 603-606. 